Doug Geisler, Eva K. Grebel, and Dante Minniti, eds.

\title{
Embedded Clusters in the Low Metallicity Galaxy SBS 0335-052
}

\author{
Leonardo Vanzi \\ ESO - European Southern Observatory, Alonso de Cordova 3107 - \\ Santiago de Chile \\ L.K. Hunt \\ CAISMI-CNR - Italy \\ T.X. Thuan \\ University of Virginia - USA
}

\begin{abstract}
We present new infrared observations of the low-metallicity blue compact galaxy SBS 0335-052. We find that the NIR emission is consistent with star formation occurring in clusters younger than $5 \mathrm{Myr}$. We detect optically hidden star formation at $4 \mu \mathrm{m}$ obscured by more than 15 mag. of visual extinction. We quantify the possible contribution from an evolved stellar population and from warm dust. We finally discuss the possible origin of the dust observed.
\end{abstract}

\section{Introduction}

SBS 0335-052 is the second lowest metallicity galaxy known after I $\mathrm{Zw} 18$. It is a Blue Compact Galaxy (BCG) hosting an exceptionally powerful episode of star formation. While the majority of BCDs show an underlying extended lowsurface-brightness component with colors indicative of an intermediate-age or old stellar population such a component has not yet been detected in SBS 0335052. Thuan et al. (1997) proposed, on the basis of HST observations, that the present burst is possibly the first star-forming episode in the history of the galaxy. According to them the star formation occurs in six Super-Star Clusters (SSCs) not older than $25 \mathrm{Myr}$ and located within a region smaller than $2^{\prime \prime}$. Thuan et al. (1999) observed with ISO a very strong continuum at $15 \mu \mathrm{m}$ that was interpreted as due to dust warmed by embedded clusters of star formation and obscured by $>15$ mags in the visual. Infrared observations are crucial to resolve the age question and to better investigate the dust in this galaxy.

\section{Observations}

We observed SBS 0335-052 in the near-infrared at the ESO-NTT and UKIRT obtaining deep and high resolution imaging and deep spectroscopy (Vanzi et al. 2000, see Fig. 1). We have also obtained new observations in the L band: a 
spectrum in the range $2.7-4.2 \mu \mathrm{m}$ with resolution $\mathrm{R}=360$ has been observed with ISAAC at the ESO-VLT (2h30m - see Fig. 1) and a 30 minutes image in the $\mathrm{L}$ band has also been obtained with the same instrument (Hunt et al. 2001).

\section{Results}

- Extinction. We have measured the extinction using the ratios of the lines $H \alpha, H \beta, B r \gamma$ and $B r \alpha$. The results are plotted in Figure 2. The hidden star formation revealed by ISO is not detected at $2 \mu \mathrm{m}$ and it only becomes observable at $4 \mu \mathrm{m}$. We detect in $\mathrm{Br} \alpha$ about 3 times more star formation than in $H \alpha$.

- Nude Clusters. The ratios of the Molecular Hydrogen lines detected in the NIR suggest fluorescent rather than thermal excitation for the $\mathrm{H}_{2}$. We can presume the $\mathrm{H}_{2}$ excitation to be related to the strong UV field that dominates SBS 0335-052 and use the ratio $(1,0) \mathrm{S}(1) / \mathrm{Br} \gamma$ as a diagnostic. From the comparison with the model of Puxley et al. (1990) we favor the excitation by large "nude" clusters of stars.

- Massive Stars. The He I lines at $1.700 \mu \mathrm{m}$ and $2.113 \mu \mathrm{m}$ can be used to constrain the presence of massive stars (Vanzi et al 1996). Both lines imply that stars with $M>35 M_{\odot}$, are present in large number.

- Young Population. The colors observed for different regions of the galaxy are plotted in Fig. 2 with SB99 (Leitherer et al. 1999 - solid line) and a model of Izotov for low metallicity $\left(Z_{\odot} / 50\right)$ stellar tracks (dotted line) plus the gas at $3 \mathrm{Myr}$ and $5 \mathrm{Myr}$ age (dark circles). The SB99 model fails to reproduce the observed colors while Izotov models reproduce well the 1-2 and 4-5 SSC colors as those of young bursts of star-formation. An old population could contribute no more than $15 \%$ of the NIR light.

- Dust. We have successfully fitted the near- and mid-infrared SED with a two-component grey body with temperatures 226 and $668 \mathrm{~K}$. Applying a starburst model to the galaxy we believe we can explain the amount of dust observed as generated by the present episode of star formation through recent supernovae.

\section{References}

Kniazev A. Y. 1997, ApJ 476, 698

Hunt L. K., Vanzi L. \& Thuan T. X. 2001, A\&A submitted

Leitherer C., Schaerer D., Goldader J. D. et al. 1999, ApJS 123, 3

Puxley P. M., Hawarden T. G. \& Mountain C. M. 1990, ApJ 364, 77

Thuan T. X., Izotov Y. I., Lipovetsky V. A. 1997, ApJ 477, 661

Thuan T. X., Sauvage M., Madden S. 1999, ApJ 516, 783

Vanzi L., Rieke G. H., Martin C. L., Shields J. C. 1996, ApJ 466, 150

Vanzi L., Hunt L. K., Thuan T. X., Izotov Y. I. 2000 A\&A 363, 493 


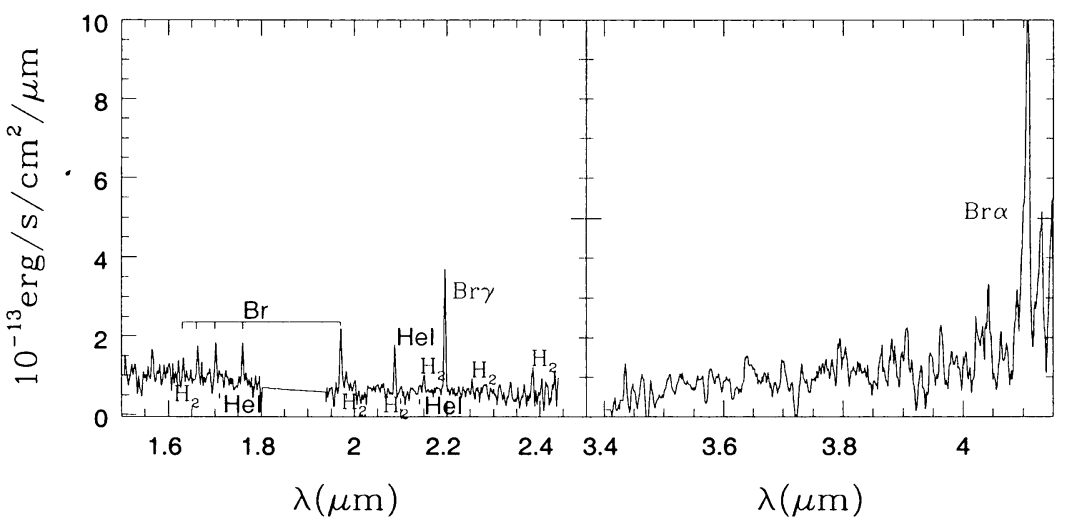

Figure 1. Near Infrared spectrum of SBS 0335-052 observed at the ESO-NTT and VLT
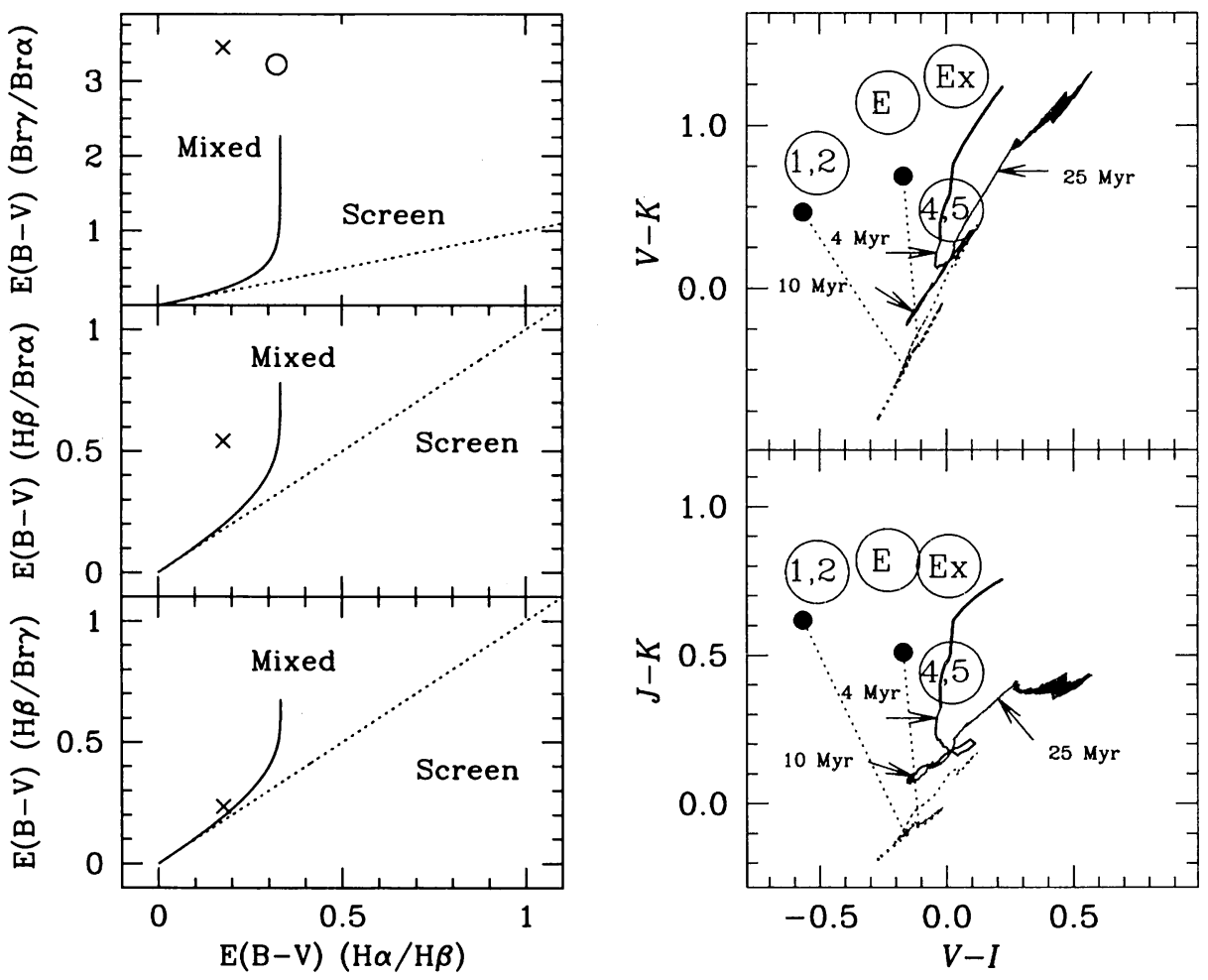

Figure 2. Color excess measured at different wavelengths (left panel). Optical-NIR color-color diagram for different regions of SBS 0335-052 plus two reference models (right panel). 